Research Article

Zheng-Qing Chu, Muhammad Salman, Asia Munir, Imran Khalid, Masood Ur Rehman*, Jia-Bao Liu, and Faisal Ali

\title{
Some topological indices of dendrimers determined by their Banhatti polynomials
}

https://doi.org/10.1515/hc-2020-0102

Received Mar 25, 2020 accepted Jul 17, 2020

\begin{abstract}
Several properties of chemical compounds in a molecular structure can be determined with the aid of mathematical languages provided by various types of topological indices. In this paper, we consider eight dendrimer structures in the context of valency based topological indices. We define four Banhatti polynomials for general (molecular) graphs, and compute them for underline dendrimers. We use these polynomials to determine four Banhatti indices. We also determine Zagreb (first, second and hyper) and forgotten indices by developing their relationships with Banhatti indices.
\end{abstract}

Keywords: Valency, topological index, Banhatti indices, Banhatti polynomials, dendrimers

AMS: 05C09; 05C92

\section{Introduction}

The structural formula of molecules and molecular compounds is represented as a molecular graph $\mathcal{N}$ in chem-

\footnotetext{
Zheng-Qing Chu, General Education Department, Anhui Xinhua University, Hefei, 230088, P.R. China, E-mail: chuzhengqing@126.com Muhammad Salman, Asia Munir, Department of Mathematics, The Islamia University of Bahawalpur, 63100, Pakistan, E-mail: muhammad.salman@iub.edu.pk, asiamuniriub1@gmail.com Imran Khalid, Faisal Ali, Center for Advanced Studies in Pure and Applied Mathematics, Bahauddin Zakariya University Multan, 60800, Pakistan, E-mails: imran.khalid@bzu.edu.pk, faisalali@bzu.edu.pk

*Corresponding Author: Masood Ur Rehman, Department of Mathematics, Abbottabad University of Science and Technology, Abbottabad, 22500, Pakistan, E-mails: masood@mail.ustc.edu.cn, masoodqau27@gmail.com

Jia-Bao Liu, School of Mathematics and Physics, Anhui Jianzhu University, Hefei, Anhui, 230601, P.R.China, E-mail:

liujiabaoad@163.com
}

ical graph theory, where nodes (vertices) are atoms and links (edges) between atoms are atom-bonds. We denote the collection of all the atoms in $\mathcal{M}$ by $A(\mathcal{M})$ and the collection of all the atom-bonds in $\mathcal{N}$ by $B(\mathcal{M})$. The number of atom-bonds in $\mathcal{M}$ is called the size of $\mathcal{M}$, and we denote it by $S(\mathcal{M})$. An atom-bond $\epsilon=a b$ between two atoms $a$ and $b$ of $\mathcal{M}$ is said to be incident with both the atoms and vice-versa, and this incidency is written as $a \epsilon$ (or $b \epsilon$ ). The valency (degree) of an atom $a$ in $\mathcal{M}$ is the number of atombonds incident with $a$, and we symbolize it as $v_{a}$. The valency of an atom-bond $\epsilon=a b \in B(\mathcal{M})$ can be computed by the formula $v_{\epsilon}=v_{a}+v_{b}-2$. Throughout this paper, the notation $\alpha \sim \beta$ will represent the type of an atom-bond whose incident atoms have valencies $\alpha$ and $\beta$, and therefore the valency of that atom-bond should be $\alpha+\beta-2$ accordingly. Whenever the size, $S(\mathcal{M})$, of a molecular graph $\mathcal{M}$ is to be found, we use the following formula provided by the well known hand shake lemma. [10]

$$
\sum_{a \in A(\mathcal{N})} v_{a}=2 S(\mathcal{N})
$$

Combinatorics and chemical graph theory play a significant role to study and characterize molecular structures, spectroscopy, and quantum chemistry $[4,7]$. In a newly developed field, called cheminformatics (a combination of chemistry, information and mathematics), qualitative structure activity/property relationships (QSAR) together with topological indices play a vital role for studying molecular compounds with chemical properties of their bioactivities. For such kind of studies, mathematicians and chemists are using several types of topological indices defined on the base of valency of atoms/atombonds, and defined on the base of distance (the number of atom-bonds lying in a shortest path) between atoms of a molecular graph. A numerical quantity which captures the symmetry of a molecular structure is known as a topological index. In fact, a topological index is a numerical characterization of a chemical graph and provides a mathematical function of the structure in QSAR studies. There are several valency based topological indices introduced to test the properties of compounds and drugs; such as 
viscosity, the gyrational radius, boiling point and many more [27]. In this paper, our aim is to study some valency based topological indices with the correlation of Banhatti indices. The valency based topological indices, which we consider, are defined as follows: In 1972, Gutman and Trinajsti [14] defined first and second Zagreb indices by the following formulae:

$Z_{1}(\mathcal{M})=\sum_{a b \in B(\mathcal{M})}\left(v_{a}+v_{b}\right)$ and $Z_{2}(\mathcal{M})=\sum_{a b \in B(\mathcal{M})}\left(v_{a} \times v_{b}\right)$.

Another novel form of Zagreb indices, called the hyper Zagreb index, were defined by Shirdel et al. [25] as follows:

$$
H Z(\mathcal{N})=\sum_{a b \in B(\mathcal{M})}\left(v_{a}+v_{b}\right)^{2} .
$$

A few years ago, in 2015, another important valency based topological index, called the forgotten index, was introduced and studied by Furtula and Gutman [12]. It can be computed by the formula

$$
F(\mathcal{M})=\sum_{a b \in B(\mathcal{M})}\left(v_{a}^{2}+v_{b}^{2}\right) .
$$

In 2016, the computation of valency based topological indices with the aid of valency of atom-bonds (edges) was initiated by Kulli [17]. He introduced four Banhatti indices, which are defined as follows:

- First and second K Banhatti indices, respectively, are:

$$
B_{1}(\mathcal{N})=\sum_{a \epsilon}\left(v_{a}+v_{\epsilon}\right) \text { and } B_{2}(\mathcal{N})=\sum_{a \epsilon}\left(v_{a} \times v_{\epsilon}\right) .
$$

- First and second K hyper Banhatti indices, respectively, are:

$$
H B_{1}(\mathcal{M})=\sum_{a \epsilon}\left(v_{a}+v_{\epsilon}\right)^{2} \text { and } H B_{2}(\mathcal{M})=\sum_{a \epsilon}\left(v_{a} \times v_{\epsilon}\right)^{2} .
$$

An extension in the theory of Banhatti indices was initiated when, in the same year 2016, Kulli defined and determined multiplicative K Banhatti indices [20]. Further extension to this theory was made in 2017 when Kulli et al. defined and computed connectivity $\mathrm{K}$ Banhatti indices including $\mathrm{K}$ harmonic Banhatti and multiplicative $\mathrm{K}$ harmonic Banhatti indices [16]. For more detail and theory about Banhatti indices, we refer the articles in $[2,5,16-$ 20, 24].

A huge amount of valency based topological indices and polynomials, defined and studied by various researchers, can be found in the literature (see $[1,3,6,8,13$, $21,22,26]$ and the references therein). In this paper, our first aim is to further extend the theory of Banhatti indices by defining four Banhatti polynomials. Moreover, we establish a few relationships of Banhatti indices, defined in (5) and (6), with the indices provided in (2), (3) and (4). Our second aim is to compute those valency based topological indices which can be determined by using the established relationships. Accordingly, we determine four Banhatti indices together with Zagreb (first, second and hyper) and forgotten indices for a few dendrimer structures with their exact values.

\section{Banhatti polynomials and some relationships}

Similar to other topological polynomials such as Mpolynomial, Zagreb polynomials, forgotten polynomial, Hosoya polynomials and many more, we define K Banhatti polynomials as follows:

- First and second K Banhatti polynomials with variable $x$, respectively, are:

$$
B_{1}(\mathcal{M}, x)=\sum_{a \epsilon} x^{\left(v_{a}+v_{\epsilon}\right)} \text { and } B_{2}(\mathcal{M}, x)=\sum_{a \epsilon} x^{\left(v_{a} \times v_{\epsilon}\right)} .
$$

- First and second $\mathrm{K}$ hyper Banhatti polynomials with variable $x$, respectively, are:

$$
H B_{1}(\mathcal{M}, x)=\sum_{a \epsilon} x^{\left(v_{a}+v_{\epsilon}\right)^{2}} \text { and } H B_{2}(\mathcal{M}, x)=\sum_{a \epsilon} x^{\left(v_{a} \times v_{\epsilon}\right)^{2}} \text {. }
$$

Clearly, by taking the first derivative of these polynomials at $x=1$, we get the corresponding Banhatti indices. Now, for a molecular graph $\mathcal{N}$, the relationships of Banhatti indices with Zagreb and forgotten indices are established as follows:

$$
\text { - } 3 Z_{1}(\mathcal{M})=B_{1}(\mathcal{N})+4 S(\mathcal{M})
$$

According to the formula of the first $\mathrm{K}$ Banhatti index from (5), we have

$$
\begin{aligned}
B_{1}(\mathcal{M}) & =\sum_{\epsilon=a b \in B(\mathcal{M})}\left[\left(v_{a}+v_{\epsilon}\right)+\left(v_{b}+v_{\epsilon}\right)\right] \\
& =\sum_{a b \in B(\mathcal{M})}\left[v_{a}+v_{b}+2\left(v_{a}+v_{b}-2\right)\right] \\
& =\sum_{a b \in B(\mathcal{M})}\left[3\left(v_{a}+v_{b}\right)-4\right] \\
& =3 Z_{1}(\mathcal{N})-4 S(\mathcal{M}) \quad \text { by using }(2)
\end{aligned}
$$

Hence, we get the required relation.

$$
\text { - } F(\mathcal{M})=B_{2}(\mathcal{M})+2\left(Z_{1}(\mathcal{M})-Z_{2}(\mathcal{M})\right)
$$


According to the formula of second $\mathrm{K}$ Banhatti index from (5), we have

$$
\begin{aligned}
B_{2}(\mathcal{N}) & =\sum_{\epsilon=a b \in B(\mathcal{N})}\left[\left(v_{a} \times v_{\epsilon}\right)+\left(v_{a} \times v_{\epsilon}\right)\right] \\
& =\sum_{a b \in B(\mathcal{N})}\left[v_{a} \times\left(v_{a}+v_{b}-2\right)+v_{b} \times\left(v_{a}+v_{b}-2\right)\right] \\
B_{2}(\mathcal{N}) & =\sum_{a b \in B(\mathcal{N})}\left[v_{a}^{2}+v_{b}^{2}+2\left(v_{a} \times v_{b}\right)-2\left(v_{a}+v_{b}\right)\right]
\end{aligned}
$$$$
B_{2}(\mathcal{M})=F(\mathcal{M})+2 Z_{2}(\mathcal{M})-2 Z_{1}(\mathcal{M}) \quad \text { by using (2) \& (4) }
$$

Hence, we get the required relation.

$$
\text { - } H Z(\mathcal{M})=B_{2}(\mathcal{M})+2 Z_{1}(\mathcal{M})
$$

From Equation (11), we have

$$
\begin{aligned}
B_{2}(\mathcal{M}) & =\sum_{a b \in B(\mathcal{M})}\left[\left(v_{a}+v_{b}\right)^{2}-2\left(v_{a}+v_{b}\right)\right] \\
& =H Z(\mathcal{M})-2 Z_{1}(\mathcal{M}) \quad \text { by using (2) \& (3) }
\end{aligned}
$$

Hence, we get the required relation.

$$
\text { - } 2\left(Z_{1}(\mathcal{N})+Z_{2}(\mathcal{N})\right)=5 B_{2}(\mathcal{N})-H B_{1}(\mathcal{N})+8 S(\mathcal{N})
$$

According to the formula of the first K hyper Banhatti index from (6), we have

$$
\begin{aligned}
& H B_{1}(\mathcal{N})=\sum_{\epsilon=a b \in B(\mathcal{N})}\left[\left(v_{a}+v_{\epsilon}\right)^{2}+\left(v_{b}+v_{\epsilon}\right)^{2}\right] \\
& =\sum_{a b \in B(\mathcal{N})}\left[\left(v_{a}+\left(v_{a}+v_{b}-2\right)\right)^{2}+\left(v_{b}+\left(v_{a}+v_{b}-2\right)\right)^{2}\right] \\
& =\sum_{a b \in B(\mathcal{N})}\left[v_{a}^{2}+v_{b}^{2}+8\left(v_{a} \times v_{b}\right)-12\left(v_{a}+v_{b}\right)+8\right]
\end{aligned}
$$

$$
H B_{1}(\mathcal{M})=5 F(\mathcal{M})+8 Z_{2}(\mathcal{M})-12 Z_{1}(\mathcal{M})+8 S(\mathcal{M})
$$$$
\text { by using (2) \& (4) }
$$

By performing the operation "Equation (15) $-5 \times$ Equation (12)”, we get the required relation.

\section{Dendrimers}

Dendrimers are molecular structures which are built symmetrically by attaching to a central core the layers of macromolecules branches with successive increments. In 1978, Buhleier at al. constructed the first dendrimers structure using the approach of synthesis divergence [9]. It has been found in the literature that dendrimers play a significant role in the fields of chemistry, biology and nanosciences [11]. Due to wide range of expediency, dendrimers attracted many researchers to investigate remarkable foundations upon them. In the context of topological indices, researchers dealt with dendrimers in the following ways (listed few): Szeged topological indices were determined by Ashrafi et al. [6]; atom-bond connectivity index was computed by Alikhani et al. [1]; M-polynomial and related indices were investigated by Munir et al. [23]; computation of forgotten polynomial and index was due to the work done by Bashir et al. [8]; and some novel valency based indices were computed by Kang et al. [15]. Attracted by these remarkable works done for dendrimers, we consider eight dendrimers, from a huge family of various types of dendrimers, in the context of valency based topological polynomials and indices defined in sections 1 and 2 . The intention to consider only these eight dendrimers is their structural symmetry and simplicity. Moreover, generations growth in these dendrimers is easily understandable to partition the atom-bonds into different parts according to their valency types, which is a necessary part to compute the underline topological indices.

\section{Poly(propyl) ether imine dendrimer}

Our first molecular graph $\mathcal{M}_{1}$ is the poly (propyl) ether imine (PETIM) dendrimer of generation $G_{\eta}$ with $\eta \geq 1$ growth stage. $\mathcal{M}_{1}$ consists of 4 branches and one central core. The central core contains 7 atoms of valency 2, 2 atoms of valency 3 and 8 atom-bonds as shown in Figure 1 . In one branch of $\mathcal{M}_{1}$, there are $8+2 \cdot 8+2^{2} \cdot 8+\ldots+2^{\eta-2} \cdot 8+$ $2^{\eta-1} \cdot 4=12 \cdot 2^{\eta-1}-8$ atoms, among them $2^{\eta-1}$ atoms are of valency $1 ; 2^{0}+2^{1}+2^{2}+\ldots+2^{\eta-1}+2^{\eta}=2 \cdot 2^{\eta}-1$ atoms are of valency 3 ; and the remaining $12 \cdot 2^{\eta-1}-8-2^{\eta-1}-2^{\eta-1}+1=$ $7 \cdot 2^{\eta-1}-7$ atoms are of valency 2 . Therefore, in $\mathcal{M}_{1}$, there are total $24 \cdot 2^{\eta}-23$ atoms; among them $2 \cdot 2^{\eta}$ atoms are of valency $1 ; 20 \cdot 2^{\eta}-21$ atoms are of valency 2 ; and $2 \cdot 2^{\eta}-2$ atoms are of valency 3 . The number of atom-bonds in $\mathcal{M}_{1}$ is $24 \cdot 2^{\eta}-24$, by formula (1). According to the valencies of atoms incident with each atom-bond, there are three types $1 \sim 2,2 \sim 2$ and $2 \sim 3$ of atom-bonds in $\mathcal{M}_{1}$. Equivalently, the atom-bonds set of $\mathcal{M}_{1}$ is partitioned as $B\left(\mathcal{M}_{1}\right)=B_{1 \sim 2} \cup B_{2 \sim 2} \cup B_{2 \sim 3}$, where

$B_{1 \sim 2}=\left\{\epsilon=a b \in B\left(\mathcal{M}_{1}\right) \mid v_{a}=1, v_{b}=2 \wedge v_{\epsilon}=1\right\}$ with $\left|B_{1 \sim 2}\right|=2 \cdot 2^{\eta}$,

$B_{2 \sim 2}=\left\{\epsilon=a b \in B\left(\mathcal{M}_{1}\right) \mid v_{a}=2, v_{b}=2 \wedge v_{\epsilon}=2\right\}$ with $\left|B_{2 \sim 2}\right|=16 \cdot 2^{\eta}-18$,

$B_{2 \sim 3}=\left\{\epsilon=a b \in B\left(\mathcal{M}_{1}\right) \mid v_{a}=2, v_{b}=3 \wedge v_{\epsilon}=3\right\}$ with $\left|B_{2 \sim 3}\right|=6 \cdot 2^{\eta}-6$. 


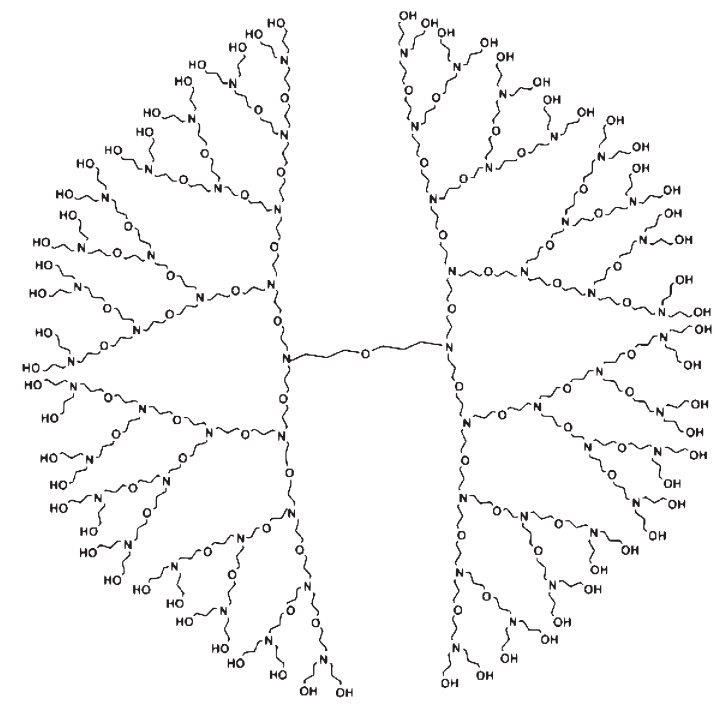

Figure 1 Poly (propyl) ether imine dendrimer

Using this partition in the formula of the first K Banhatti polynomial from (7), we have:

$$
\begin{aligned}
B_{1}\left(\mathcal{M}_{1}, x\right) & =\left(\sum_{\epsilon=a b \in B_{1 \sim 2}}+\sum_{\epsilon=a b \in B_{2 \sim 2}}+\sum_{\epsilon=a b \in B_{2 \sim 3}}\right) x^{\left(v_{a}+v_{\epsilon}\right)} \\
& =2 \cdot 2^{\eta} x^{5}+\left(16 \cdot 2^{\eta}-18\right) x^{8}+\left(6 \cdot 2^{\eta}-6\right) x^{11} .
\end{aligned}
$$

Analogously, using the atom-bonds partition in formulae of the other three Banhatti polynomials from (7) and (8), it can be found that

$$
\begin{aligned}
B_{2}\left(\mathcal{M}_{1}, x\right) & =2 \cdot 2^{\eta} x^{3}+\left(16 \cdot 2^{\eta}-18\right) x^{8}+\left(6 \cdot 2^{\eta}-6\right) x^{15}, \\
H B_{1}\left(\mathcal{M}_{1}, x\right) & =2 \cdot 2^{\eta} x^{13}+\left(16 \cdot 2^{\eta}-18\right) x^{32}+\left(6 \cdot 2^{\eta}-6\right) x^{61}, \\
H B_{2}\left(\mathcal{M}_{1}, x\right) & =2 \cdot 2^{\eta} x^{5}+\left(16 \cdot 2^{\eta}-18\right) x^{32}+\left(6 \cdot 2^{\eta}-6\right) x^{117} .
\end{aligned}
$$

- Figure 2 poly ethylene amido amine dendrimer

With respect to $x$, by taking the first derivative of these polynomials at $x=1$, we get the following K Banhatti indices of $\mathcal{M}_{1}$.

$\operatorname{TI}\left(\mathcal{M}_{1}\right)=\left\{\begin{array}{ccl}204 \cdot 2^{\eta}-210 & , & \text { when } T I \text { is } B_{1}, \\ 224 \cdot 2^{\eta}-234 & , & \text { when } T I \text { is } B_{2}, \\ 904 \cdot 2^{\eta}-942 & , & \text { when } T I \text { is } H B_{1}, \\ 1224 \cdot 2^{\eta}-1278 & , & \text { when } T I \text { is } H B_{2} .\end{array}\right.$

Using relationships (9), (10), (13) and (14), we get the following Zagreb and forgotten indices of $\mathcal{M}_{1}$.

$\operatorname{TI}\left(\mathcal{M}_{1}\right)=\left\{\begin{array}{lll}100 \cdot 2^{\eta}-102 & , & \text { when } T I \text { is } Z_{1}, \\ 104 \cdot 2^{\eta}-108 & , & \text { when } T I \text { is } Z_{2}, \\ 424 \cdot 2^{\eta}-438 & , & \text { when } T I \text { is } H Z, \\ 216 \cdot 2^{\eta}-222 & , & \text { when } T I \text { is } F .\end{array}\right.$

The value for the forgotten index $F$ is similar with its value found by Bashir et al. in [8].

\section{Poly ethylene amido amine dendrimer}

Our second molecular graph $\mathcal{M}_{2}$ is the poly ethylene amido amine (PETAA) dendrimer of generation $G_{\eta}$ with $\eta \geq 1$ growth stage. $\mathcal{M}_{2}$ consists of 4 branches and one central core. The central core contains 2 atoms of valency 1,8 atoms of valency 2, 4 atoms of valency 3, and 13 atombonds as depicted in Figure 2. In one branch of $\mathcal{M}_{2}$, there are $8+2 \cdot 8+2^{2} \cdot 8+\ldots+2^{\eta-1} \cdot 8+2^{\eta} \cdot 3=11 \cdot 2^{\eta}-8$ atoms, among them $2^{0}+2^{1}+2^{2}+\ldots+2^{\eta-1}+2^{\eta}=2 \cdot 2^{\eta}-1$ are leaves (atoms with valency 1 ); $5+2 \cdot 5+2^{2} \cdot 5+\ldots+2^{\eta-1} \cdot 5+$ $2^{\eta+1}=7 \cdot 2^{\eta}-5$ atoms are of valency 2 ; and the remaining $11 \cdot 2^{\eta}-8-2 \cdot 2^{\eta}+1-7 \cdot 2 m+5=2 \cdot 2^{\eta}-2$ atoms are of valency 3 . Thus $\mathcal{M}_{2}$ has total $44 \cdot 2^{\eta}-18$ atoms, among them $8 \cdot 2^{\eta}-2$ atoms are of valency $1 ; 28 \cdot 2^{\eta}-12$ atoms are of valency 2 ; and $8 \cdot 2^{\eta}-4$ atoms are of valency 3 .

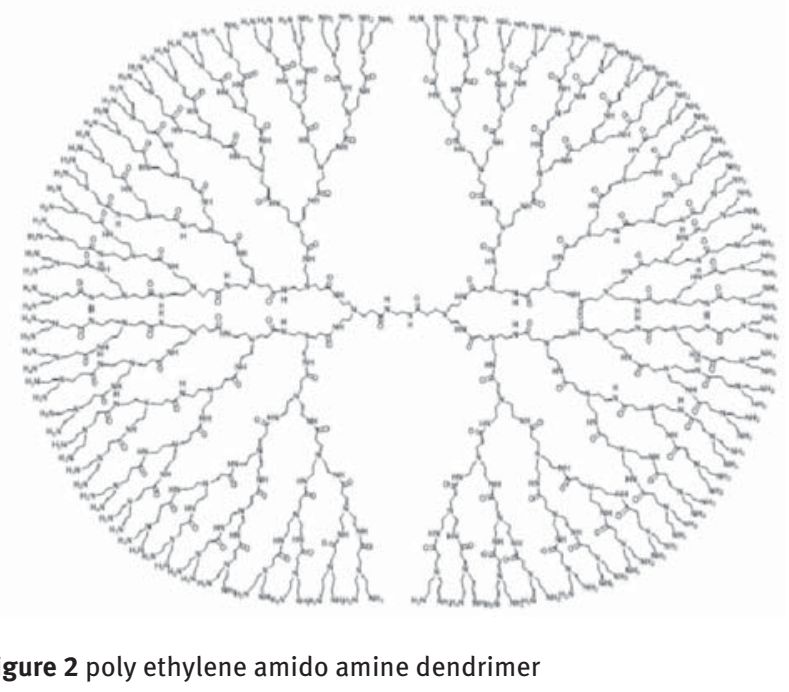

The number of atom-bonds in $\mathcal{M}_{2}$ is $44 \cdot 2^{\eta}-19$, by formula (1). With respect to the valencies of atoms incident with each atom-bond, there are four types $1 \sim 2,1 \sim 3$, $2 \sim 2$ and $2 \sim 3$ of atom-bonds in $\mathcal{M}_{2}$. Equivalently, the atom-bonds set of $\mathcal{M}_{2}$ is partitioned as $B\left(\mathcal{M}_{2}\right)=B_{1 \sim 2} U$ $B_{1 \sim 3} \cup B_{2 \sim 2} \cup B_{2 \sim 3}$, where

$B_{1 \sim 2}=\left\{\epsilon=a b \in B\left(\mathcal{M}_{2}\right) \mid v_{a}=1, v_{b}=2 \wedge v_{\epsilon}=1\right\}$ with $\left|B_{1 \sim 2}\right|=4 \cdot 2^{\eta}$,

$B_{1 \sim 3}=\left\{\epsilon=a b \in B\left(\mathcal{M}_{2}\right) \mid v_{a}=1, v_{b}=3 \wedge v_{\epsilon}=2\right\}$ with $\left|B_{1 \sim 3}\right|=4 \cdot 2^{\eta}-2$,

$B_{2 \sim 2}=\left\{\epsilon=a b \in B\left(\mathcal{M}_{2}\right) \mid v_{a}=2, v_{b}=2 \wedge v_{\epsilon}=2\right\}$ with $\left|B_{2 \sim 2}\right|=16 \cdot 2^{\eta}-7$,

$B_{2 \sim 3}=\left\{\epsilon=a b \in B\left(\mathcal{M}_{2}\right) \mid v_{a}=2, v_{b}=3 \wedge v_{\epsilon}=3\right\}$ with $\left|B_{2 \sim 3}\right|=20 \cdot 2^{\eta}-10$.

Using this partition in the formula of the first $\mathrm{K}$ Banhatti 
polynomial from (7), we have:

$$
\begin{aligned}
& B_{1}\left(\mathcal{M}_{2}, x\right) \\
& =\left(\sum_{\epsilon=a b \in B_{1 \sim 2}}+\sum_{\epsilon=a b \in B_{1 \sim 3}}+\sum_{\epsilon=a b \in B_{2 \sim 2}}+\sum_{\epsilon=a b \in B_{2 \sim 3}}\right) x^{\left(v_{a}+v_{\epsilon}\right)} \\
& =4 \cdot 2^{\eta} x^{5}+\left(20 \cdot 2^{\eta}-9\right) x^{8}+\left(20 \cdot 2^{\eta}-10\right) x^{11} .
\end{aligned}
$$

Equivalently, using the atom-bonds partition in formulae of the other three Banhatti polynomials from (7) and (8), it can be found that

$$
\begin{aligned}
B_{2}\left(\mathcal{M}_{2}, x\right)= & \cdot \cdot 2^{\eta} x^{3}+\left(20 \cdot 2^{\eta}-9\right) x^{8} \\
& +\left(20 \cdot 2^{\eta}-10\right) x^{15} \\
H B_{1}\left(\mathcal{M}_{2}, x\right)= & 4 \cdot 2^{\eta} x^{13}+\left(4 \cdot 2^{\eta}-2\right) x^{34}+\left(16 \cdot 2^{\eta}-7\right) x^{32} \\
& +\left(20 \cdot 2^{\eta}-10\right) x^{61} \\
H B_{2}\left(\mathcal{M}_{2}, x\right)= & 4 \cdot 2^{\eta} x^{5}+\left(4 \cdot 2^{\eta}-2\right) x^{40}+\left(16 \cdot 2^{\eta}-7\right) x^{32} \\
& +\left(20 \cdot 2^{\eta}-10\right) x^{117} .
\end{aligned}
$$

With respect to $x$, by taking the first derivative of these polynomials at $x=1$, we get the following K Banhatti indices of $\mathcal{M}_{2}$

$\operatorname{TI}\left(\mathcal{M}_{2}\right)=\left\{\begin{array}{ccl}400 \cdot 2^{\eta}-182 & , & \text { when } T I \text { is } B_{1}, \\ 472 \cdot 2^{\eta}-222 & , & \text { when } T I \text { is } B_{2}, \\ 1920 \cdot 2^{\eta}-902 & , & \text { when } T I \text { is } H B_{1}, \\ 3032 \cdot 2^{\eta}-1474 & , & \text { when } T I \text { is } H B_{2} .\end{array}\right.$

Using relationships (9), (10), (13) and (14), we have the following Zagreb and forgotten indices of $\mathcal{M}_{2}$.

$\operatorname{TI}\left(\mathcal{M}_{2}\right)=\left\{\begin{array}{ccl}192 \cdot 2^{\eta}-86 & , & \text { when } T I \text { is } Z_{1}, \\ 204 \cdot 2^{\eta}-94 & , & \text { when } T I \text { is } Z_{2}, \\ 856 \cdot 2^{\eta}-394 & , & \text { when } T I \text { is } H Z, \\ 448 \cdot 2^{\eta}-206 & , & \text { when } T I \text { is } F .\end{array}\right.$

\section{Porphyrin dendrimer}

Our third molecular graph $\mathcal{M}_{3}$ is the porphyrin dendrimer ( $D_{\kappa} P_{\kappa}$ ) of generation $G_{\kappa}$ with $\kappa=2^{\eta}$ growth stage for $\eta \geq 2$. The structure of $\mathcal{M}_{3}$ consists of 4 branches and one central core. The central core contains 4 atoms of valency 2 , 2 atoms of valency 3, and 5 atom-bonds as shown in Figure 3. In one branch of $\mathcal{M}_{3}$, there are $4+2 \cdot 4+2^{2} \cdot 4+$ $\ldots+2^{\eta-3} \cdot 4+2^{\eta-2} \cdot 92=96 \cdot 2^{\eta-2}-4$ atoms, among them $26 \cdot 2^{\eta-2}$ atoms are of valency $1 ; 3+2 \cdot 3+2^{2} \cdot 3+$ $\ldots+2^{\eta-3} \cdot 3+2^{\eta-2} \cdot 31=34 \cdot 2^{\eta-2}-3$ atoms are of valency $2 ; 8 \cdot 2^{\eta-2}$ atoms are of valency 4 ; and the remaining $96 \cdot 2^{\eta-2}-4-26 \cdot 2^{\eta-2}-34 \cdot 2^{\eta-2}+3-8 \cdot 2^{\eta-2}=28 \cdot 2^{\eta-2}-1$ atoms are of valency 3 . So $\mathcal{M}_{3}$ keeps total $96 \cdot 2^{\eta}-10$ atoms, among them $26 \cdot 2^{\eta}$ atoms are of valency $1 ; 34 \cdot 2^{\eta}-8$ atoms are of valency $2 ; 28 \cdot 2^{\eta}-2$ atoms are of valency 3 ; and the remaining $8 \cdot 2^{\eta}$ atoms are of valency 4 .

The number of atom-bonds in $\mathcal{M}_{3}$ is $105 \cdot 2^{\eta}-11$, by formula (1). According to the valencies of atoms incident with each atom-bond, there are six types $1 \sim 3,1 \sim 4$, $2 \sim 2,2 \sim 3,3 \sim 3$, and $3 \sim 4$ of atom-bonds in $\mathcal{M}_{3}$. Equivalently, the partition of atom-bonds set of $\mathcal{M}_{3}$ is $B\left(\mathcal{M}_{3}\right)=B_{1 \sim 3} \cup B_{1 \sim 4} \cup B_{2 \sim 2} \cup B_{2 \sim 3} \cup B_{3 \sim 3} \cup B_{3 \sim 4}$, where $B_{1 \sim 3}=\left\{\epsilon=a b \in B\left(\mathcal{M}_{3}\right) \mid v_{a}=1, v_{b}=3 \wedge v_{\epsilon}=2\right\}$ with $\left|B_{1 \sim 3}\right|=2 \cdot 2^{\eta}$,

$B_{1 \sim 4}=\left\{\epsilon=a b \in B\left(\mathcal{M}_{3}\right) \mid v_{a}=1, v_{b}=4 \wedge v_{\epsilon}=3\right\}$ with $\left|B_{1 \sim 4}\right|=24 \cdot 2^{\eta}$,

$B_{2 \sim 2}=\left\{\epsilon=a b \in B\left(\mathcal{M}_{3}\right) \mid v_{a}=2, v_{b}=2 \wedge v_{\epsilon}=2\right\}$ with $\left|B_{2 \sim 2}\right|=10 \cdot 2^{\eta}-5$,

$B_{2 \sim 3}=\left\{\epsilon=a b \in B\left(\mathcal{M}_{3}\right) \mid v_{a}=2, v_{b}=3 \wedge v_{\epsilon}=3\right\}$ with $\left|B_{2 \sim 3}\right|=48 \cdot 2^{\eta}-6$,

$B_{3 \sim 3}=\left\{\epsilon=a b \in B\left(\mathcal{M}_{3}\right) \mid v_{a}=3, v_{b}=3 \wedge v_{\epsilon}=4\right\}$ with $\left|B_{3 \sim 3}\right|=13 \cdot 2^{\eta}$,

$B_{3 \sim 4}=\left\{\epsilon=a b \in B\left(\mathcal{M}_{3}\right) \mid v_{a}=3, v_{b}=4 \wedge v_{\epsilon}=5\right\}$ with $\left|B_{3 \sim 4}\right|=8 \cdot 2^{\eta}$.

Using this partition in the formula of the first $\mathrm{K}$ Banhatti polynomial from (7), we have:

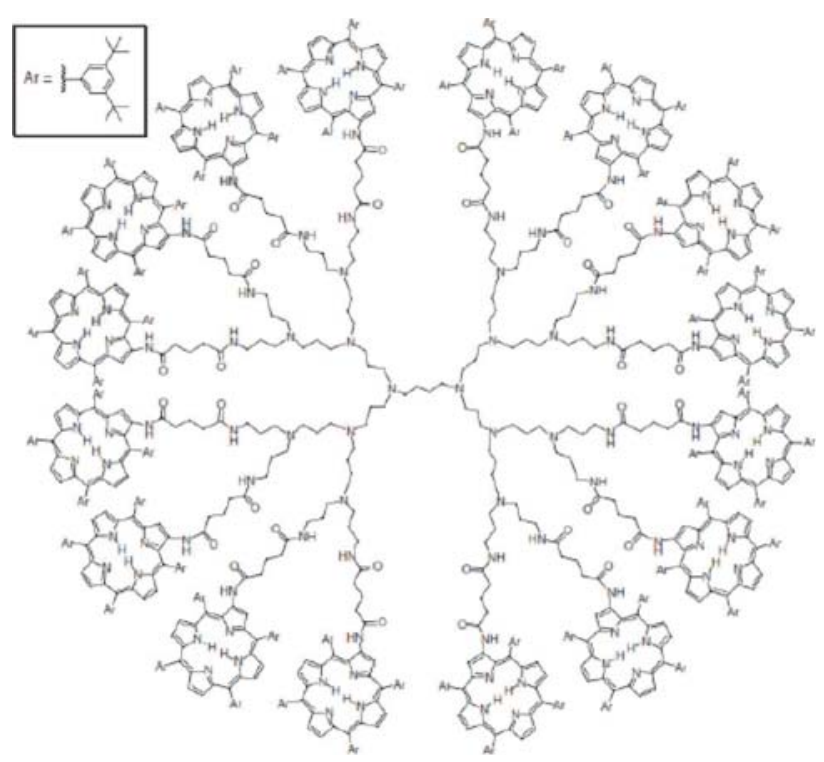

Figure 3 Porphyrin dendrimer

$$
\begin{aligned}
& B_{1}\left(\mathcal{M}_{3}, x\right)=\left(\sum_{\epsilon=a b \in B_{1 \sim 3}}+\sum_{\epsilon=a b \in B_{1 \sim 4}}+\sum_{\epsilon=a b \in B_{2 \sim 2}}\right. \\
& \left.+\sum_{\epsilon=a b \in B_{2 \sim 3}}+\sum_{\epsilon=a b \in B_{3 \sim 3}}+\sum_{\epsilon=a b \in B_{3 \sim 4}}\right) x^{\left(v_{a}+v_{\epsilon}\right)}
\end{aligned}
$$


$=\left(12 \cdot 2^{\eta}-5\right) x^{8}+\left(72 \cdot 2^{\eta}-6\right) x^{11}+13 \cdot 2^{\eta} x^{14}+8 \cdot 2^{\eta} x^{17}$.

Similarly, using the atom-bonds partition in formulae of the other three Banhatti polynomials from (7) and (8), it can be found that

$$
\begin{aligned}
B_{2}\left(\mathcal{M}_{3}, x\right)= & \left(12 \cdot 2^{\eta}-5\right) x^{8}+\left(72 \cdot 2^{\eta}-6\right) x^{15} \\
& +13 \cdot 2^{\eta} x^{24}+8 \cdot 2^{\eta} x^{35}, \\
H B_{1}\left(\mathcal{M}_{3}, x\right)= & \left(10 \cdot 2^{\eta}-5\right) x^{32}+2 \cdot 2^{\eta} x^{34}+\left(48 \cdot 2^{\eta}-6\right) x^{61} \\
& +8 \cdot 2^{\eta} x^{65}+13 \cdot 2^{\eta} x^{98}+8 \cdot 2^{\eta} x^{145}, \\
H B_{2}\left(\mathcal{M}_{3}, x\right)= & \left(10 \cdot 2^{\eta}-5\right) x^{32}+2 \cdot 2^{\eta} x^{40}+\left(48 \cdot 2^{\eta}-6\right) x^{117} \\
& +24 \cdot 2^{\eta} x^{153}+13 \cdot 2^{\eta} x^{288}+8 \cdot 2^{\eta} x^{625} .
\end{aligned}
$$

With respect to $x$, by taking the first derivative of these polynomials at $x=1$, we get the following K Banhatti indices of $\mathcal{M}_{3}$.

$\operatorname{TI}\left(\mathcal{M}_{3}\right)=\left\{\begin{array}{cll}1206 \cdot 2^{\eta}-106 & , & \text { when } T I \text { is } B_{1}, \\ 1768 \cdot 2^{\eta}-130 & , & \text { when } T I \text { is } B_{2}, \\ 7470 \cdot 2^{\eta}-526 & , & \text { when } T I \text { is } H B_{1}, \\ 19218 \cdot 2^{\eta}-862 & , & \text { when } T I \text { is } H B_{2} .\end{array}\right.$

Using relationships (9), (10), (13) and (14), Zagreb and forgotten indices of $\mathcal{M}_{3}$ are:

$\operatorname{TI}\left(\mathcal{M}_{3}\right)=\left\{\begin{array}{ccl}542 \cdot 2^{\eta}-50 & , & \text { when } T I \text { is } Z_{1}, \\ 643 \cdot 2^{\eta}-56 & , & \text { when } T I \text { is } Z_{2}, \\ 2852 \cdot 2^{\eta}-230 & , & \text { when } T I \text { is } H Z, \\ 1566 \cdot 2^{\eta}-118 & , & \text { when } T I \text { is } F .\end{array}\right.$

The value for the forgotten index is similar with its value determined by Bashir et al. in [8].

\section{Zinc porphyrin dendrimer}

Our fourth molecular graph $\mathcal{M}_{4}$ is the zinc porphyrin dendrimer $\left(D P Z_{m}\right)$ of generation $G_{\eta}$ with $\eta \geq 1$ growth stage. $\mathcal{M}_{4}$ consists of 4 branches and one central core. The central core contains 24 atoms of valencies 2 and 3, 1 atom of valency 4, and 60 atom-bonds as displayed in Figure 4. In one branch of $\mathcal{M}_{4}$, we have $14+2 \cdot 14+2^{2} \cdot 14+\ldots+2^{\eta-1} \cdot 14=14\left(2^{\eta}-1\right)$ atoms, among them $9+2 \cdot 9+2^{2} \cdot 9+\ldots+2^{\eta-1} \cdot 9+2^{\eta-1} \cdot 11=$ $20 \cdot 2^{\eta-1}-9$ atoms are of valency 2 , and the remaining $14\left(2^{\eta}-1\right)-20 \cdot 2^{\eta-1}+9=4 \cdot 2^{\eta}-5$ atoms are of valency 3 . Therefore, there are total $56 \cdot 2^{\eta}-7$ atoms in $\mathcal{M}_{4}$, among them $40 \cdot 2^{\eta}-12$ atoms are of valency $2,16 \cdot 2^{\eta}+4$ atoms are of valency 3 , and one atom is of valency 4. The number of atom-bonds in $\mathcal{M}_{4}$ is $64 \cdot 2^{\eta}-4$, by formula (1). According to the valencies of atoms incident with each atom-bond, there are four types

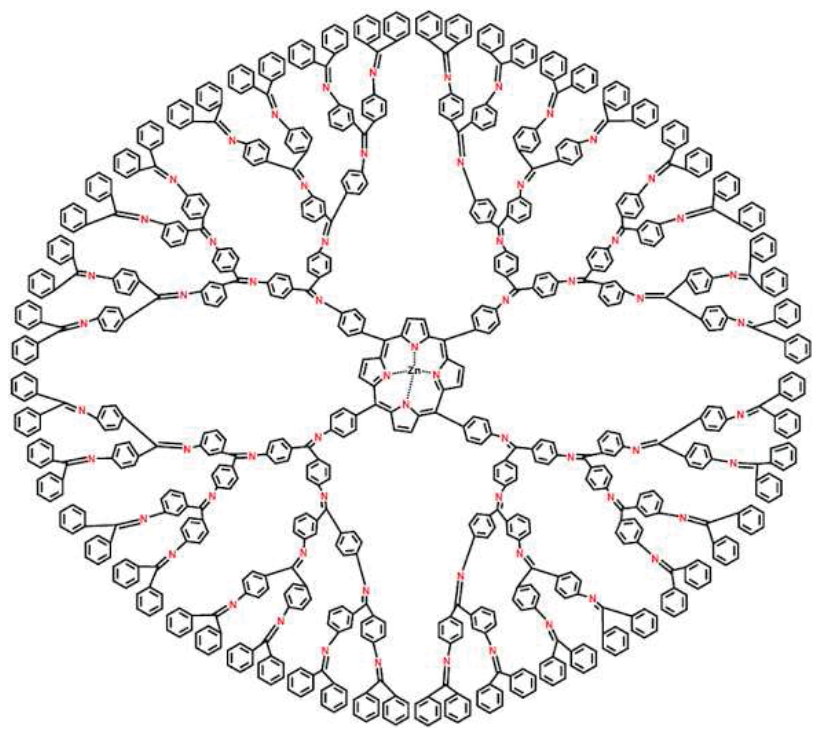

Figure 4 Zinc porphyrin dendrimer

$2 \sim 2,2 \sim 3,3 \sim 3$, and $3 \sim 4$ of atom-bonds in $\mathcal{M}_{4}$. Accordingly, the atom-bonds set of $\mathcal{M}_{4}$ is partitioned as $B\left(\mathcal{M}_{4}\right)=B_{2 \sim 2} \cup B_{2 \sim 3} \cup B_{3 \sim 3} \cup B_{3 \sim 4}$, where

$B_{2 \sim 2}=\left\{\epsilon=a b \in B\left(\mathcal{M}_{4}\right) \mid v_{a}=2, v_{b}=2 \wedge v_{\epsilon}=2\right\}$ with $\left|B_{2 \sim 2}\right|=16 \cdot 2^{\eta}-4$,

$B_{2 \sim 3}=\left\{\epsilon=a b \in B\left(\mathcal{M}_{4}\right) \mid v_{a}=2, v_{b}=3 \wedge v_{\epsilon}=3\right\}$ with $\left|B_{2 \sim 3}\right|=40 \cdot 2^{\eta}-16$,

$B_{3 \sim 3}=\left\{\epsilon=a b \in B\left(\mathcal{M}_{4}\right) \mid v_{a}=3, v_{b}=3 \wedge v_{\epsilon}=4\right\}$ with $\left|B_{3 \sim 3}\right|=8 \cdot 2^{\eta}+12$,

$B_{3 \sim 4}=\left\{\epsilon=a b \in B\left(\mathcal{M}_{4}\right) \mid v_{a}=3, v_{b}=4 \wedge v_{\epsilon}=5\right\}$ with $\left|B_{3 \sim 4}\right|=4$.

Using this partition in the formula of the first K Banhatti polynomial from (7), we have:

$$
\begin{aligned}
B_{1}\left(\mathcal{M}_{4}, x\right)= & \left(\sum_{\epsilon=a b \in B_{2 \sim 2}}+\sum_{\epsilon=a b \in B_{2 \sim 3}}+\sum_{\epsilon=a b \in B_{3} \sim 3}\right. \\
& \left.+\sum_{\epsilon=a b \in B_{3 \sim 4}}\right) x^{\left(v_{a}+v_{\epsilon}\right)} \\
= & \left(16 \cdot 2^{\eta}-4\right) x^{8}+\left(40 \cdot 2^{\eta}-16\right) x^{11} \\
& +\left(8 \cdot 2^{\eta}+12\right) x^{12}+4 x^{17} .
\end{aligned}
$$

On the same way, using the atom-bonds partition in formulae of the other Banhatti polynomials align (7) and (8), it can be found that

$$
\begin{array}{cc}
B_{2}\left(\mathcal{M}_{4}, x\right)= & \left(16 \cdot 2^{\eta}-4\right) x^{8}+\left(40 \cdot 2^{\eta}-16\right) x^{15} \\
& +\left(8 \cdot 2^{\eta}+12\right) x^{18}+4 x^{35}, \\
H B_{1}\left(\mathcal{M}_{4}, x\right)= & \left(16 \cdot 2^{\eta}-4\right) x^{32}+\left(40 \cdot 2^{\eta}-16\right) x^{61} \\
& +\left(8 \cdot 2^{\eta}+12\right) x^{72}+4 x^{145},
\end{array}
$$




$$
\begin{gathered}
H B_{2}\left(\mathcal{M}_{4}, x\right)=\left(16 \cdot 2^{\eta}-4\right) x^{32}+\left(40 \cdot 2^{\eta}-16\right) x^{117} \\
+\left(8 \cdot 2^{\eta}+12\right) x^{162}+4 x^{625} .
\end{gathered}
$$

With respect to $x$, by taking the first derivative of these polynomials at $x=1$, we get the following K Banhatti indices of $\mathcal{M}_{4}$.

$\operatorname{TI}\left(\mathcal{M}_{4}\right)=\left\{\begin{array}{ccl}664 \cdot 2^{\eta}+4 & , & \text { when } T I \text { is } B_{1}, \\ 872 \cdot 2^{\eta}+84 & , & \text { when } T I \text { is } B_{2}, \\ 3528 \cdot 2^{\eta}+340 & , & \text { when } T I \text { is } H B_{1}, \\ 6488 \cdot 2^{\eta}+2444 & , & \text { when } T I \text { is } H B_{2} .\end{array}\right.$

Using relationships (9), (10), (13) and (14), we receive the Zagreb and forgotten indices of $\mathcal{M}_{4}$ as follows:

$\operatorname{TI}\left(\mathcal{M}_{4}\right)=\left\{\begin{array}{ccl}307 \cdot 2^{\eta}-4 & , & \text { when } T I \text { is } Z_{1}, \\ 341 \cdot 2^{\eta}-7 & , & \text { when } T I \text { is } Z_{2}, \\ 260 \cdot 2^{\eta}+108 & , & \text { when } T I \text { is } H Z, \\ 756 \cdot 2^{\eta}+20 & , & \text { when } T I \text { is } F .\end{array}\right.$

\section{Porphyrin cored dendrimers}

Porphyrin cored dendrimers are of four types. Accordingly, we consider four subsections.

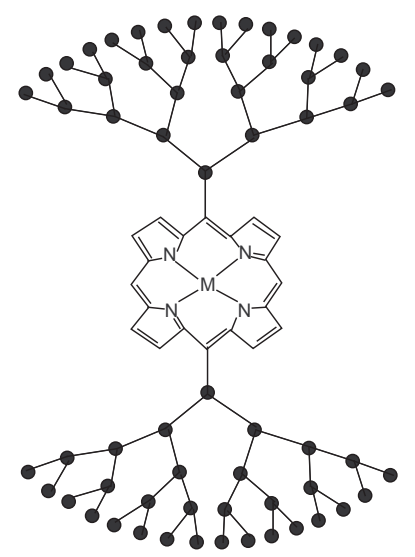

Figure 5 Porphyrin cored dendrimer-I

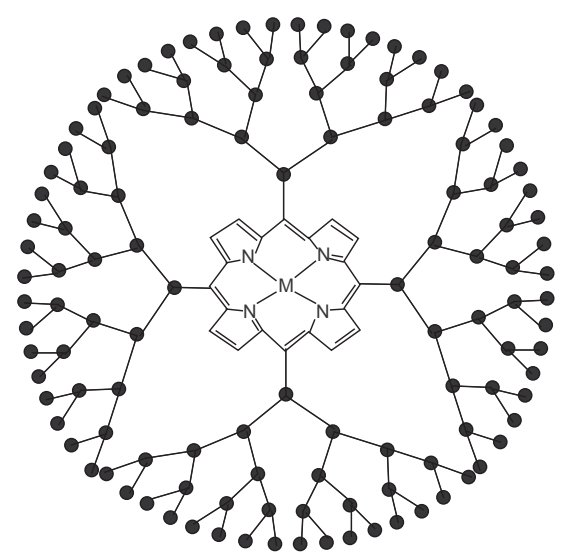

Figure 6 Porphyrin cored dendrimer-II

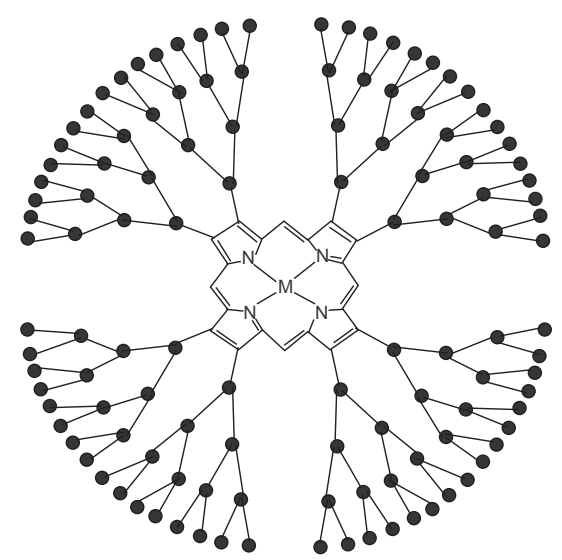

Figure 7 Porphyrin cored dendrimer-III

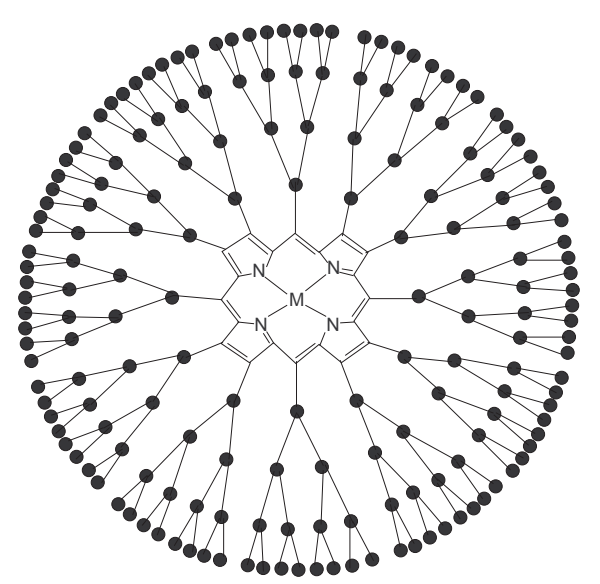

Figure 8 Porphyrin cored dendrimer-IV 


\section{Porphyrin cored dendrimers-I}

Fifth molecular graph $\mathcal{M}_{51}$ considered in this paper is the porphyrin cored dendrimer-I (PCD-I) of generation $G_{\eta}$ with $\eta \geq 1$ growth stage. $\mathcal{M}_{51}$ consists of two similar branches and one central core. The central core contains 10 atoms of valency 2, 14 atoms of valency 3, 1 atom of valency 4, and 34 atom-bonds as shown in Figure 5. In one branch of $\mathcal{M}_{51}$, there are $2^{0}+2^{1}+2^{2}+\ldots+2^{\eta}=2^{\eta+1}-1$ atoms, among them $2^{\eta}$ atoms are of valency 1 , and $2^{0}+2^{1}+2^{2}+\ldots+2^{\eta-1}=2^{\eta}-1$ atoms are of valency 3 . Therefore, in $\mathcal{M}_{51}$, there are total $4 \cdot 2^{\eta}+23$ atoms, among them $2 \cdot 2^{\eta}$ atoms are of valency 1 , $2 \times 2^{\eta}+12$ atoms are of valency 3,10 atoms are of valency 2 , and one atom is of valency 4 .

The number of atom-bonds in $\mathcal{M}_{51}$ is $4 \cdot 2^{\eta}+30$, by formula (1). According to the valencies of atoms incident with each atom-bond, there are five types $1 \sim 3$, $2 \sim 2,2 \sim 3,3 \sim 3$, and $3 \sim 4$ of atom-bonds in $\mathcal{M}_{51}$. Equivalently, the atom-bonds set of $\mathcal{M}_{51}$ is partitioned as $B\left(\mathcal{M}_{51}\right)=B_{1 \sim 3} \cup B_{2 \sim 2} \cup B_{2 \sim 3} \cup B_{3 \sim 3} \cup B_{3 \sim 4}$, where $B_{1 \sim 3}=\left\{\epsilon=a b \in B\left(\mathcal{M}_{51}\right) \mid v_{a}=1, v_{b}=3 \wedge v_{\epsilon}=2\right\}$ with $\left|B_{1 \sim 3}\right|=2 \cdot 2^{\eta}$,

$B_{2 \sim 2}=\left\{\epsilon=a b \in B\left(\mathcal{M}_{51}\right) \mid v_{a}=2, v_{b}=2 \wedge v_{\epsilon}=2\right\}$ with $\left|B_{2 \sim 2}\right|=4$,

$B_{2 \sim 3}=\left\{\epsilon=a b \in B\left(\mathcal{M}_{51}\right) \mid v_{a}=2, v_{b}=3 \wedge v_{\epsilon}=3\right\}$ with $\left|B_{2 \sim 3}\right|=12$,

$B_{3 \sim 3}=\left\{\epsilon=a b \in B\left(\mathcal{M}_{51}\right) \mid v_{a}=3, v_{b}=3 \wedge v_{\epsilon}=4\right\}$ with $\left|B_{3 \sim 3}\right|=2 \cdot 2^{\eta}+10$,

$B_{3 \sim 4}=\left\{\epsilon=a b \in B\left(\mathcal{M}_{51}\right) \mid v_{a}=3, v_{b}=4 \wedge v_{\epsilon}=5\right\}$ with $\left|B_{3 \sim 4}\right|=4$.

Using this partition in the formula of the first K Banhatti polynomial from (7), we have:

$$
\begin{aligned}
B_{1}\left(\mathcal{M}_{51}, x\right)= & \left(\sum_{\epsilon=a b \in B_{1 \sim 3}}+\sum_{\epsilon=a b \in B_{2 \sim 2}}+\sum_{\epsilon=a b \in B_{2 \sim 3}}+\sum_{\epsilon=a b \in B_{3 \sim 3}}\right. \\
& \left.+\sum_{\epsilon=a b \in B_{3 \sim 4}}\right) x^{\left(v_{a}+v_{\epsilon}\right)} \\
= & \left(2 \cdot 2^{\eta}+4\right) x^{8}+12 x^{11}+\left(2 \cdot 2^{\eta}+10\right) x^{14} \\
& +4 x^{17} .
\end{aligned}
$$

Analogously, using the atom-bonds partition in formulae of the other three Banhatti polynomials from (7) and (8), it can be found that

$$
\begin{aligned}
B_{2}\left(\mathcal{M}_{51}, x\right)= & 2 \cdot 2^{\eta} x^{8}+4 x^{8}+12 x^{15} \\
& +\left(2 \cdot 2^{\eta}+10\right) x^{24}+4 x^{35}, \\
H B_{1}\left(\mathcal{M}_{51}, x\right)= & 2 \cdot 2^{\eta} x^{34}+8 x^{16}+12 x^{61} \\
& +\left(2 \cdot 2^{\eta}+10\right) x^{98}+4 x^{145}, \\
H B_{2}\left(\mathcal{M}_{51}, x\right)= & 2 \cdot 2^{\eta} x^{40}+8 x^{16}+12 x^{117}
\end{aligned}
$$

$$
+\left(2 \cdot 2^{\eta}+10\right) x^{288}+4 x^{625} \text {. }
$$

With respect to $x$, by taking the first derivative of these polynomials at $x=1$, we get the following $\mathrm{K}$ Banhatti indices of $\mathcal{M}_{51}$.

$\operatorname{TI}\left(\mathcal{M}_{51}\right)=\left\{\begin{array}{cll}44 \cdot 2^{\eta}+372 & , & \text { when } T I \text { is } B_{1}, \\ 64 \cdot 2^{\eta}+430 & , & \text { when } T I \text { is } B_{2}, \\ 264 \cdot 2^{\eta}+2420 & , & \text { when } T I \text { is } H B_{1}, \\ 656 \cdot 2^{\eta}+6912 & , & \text { when } T I \text { is } H B_{2}\end{array}\right.$

Using relationships (9), (10), (13) and (14), Zagreb and forgotten indices of $\mathcal{M}_{51}$ have the following values:

$\operatorname{TI}\left(\mathcal{M}_{51}\right)=\left\{\begin{array}{cll}20 \cdot 2^{\eta}+164 & , & \text { when } T I \text { is } Z_{1}, \\ 24 \cdot 2^{\eta}+226 & , & \text { when } T I \text { is } Z_{2}, \\ 104 \cdot 2^{\eta}+920 & , & \text { when } T I \text { is } H Z, \\ 56 \cdot 2^{\eta}+468 & , & \text { when } T I \text { is } F .\end{array}\right.$

\section{Porphyrin cored dendrimer-II}

Our sixth molecular graph $\mathcal{M}_{52}$ is the porphyrin cored dendrimer-II (PCD-II) of generation $G_{\eta}$ with $\eta \geq 1$ growth stage. $\mathcal{M}_{52}$ consists of four similar branches and one central core. The central core contains 8 atoms of valency 2 , 16 atoms of valency 3, 1 atom of valency 4, and 36 atombonds as shown in Figure 6. In one branch of $\mathcal{M}_{52}$, there are $2^{0}+2^{1}+2^{2}+\ldots+2^{\eta}=2^{\eta+1}-1$ atoms, among them $2^{\eta}$ atoms are of valency 1 , and $2^{0}+2^{1}+2^{2}+\ldots+2^{\eta-1}=2^{\eta}-1$ atoms are of valency 3 . Therefore, in $\mathcal{M}_{52}$, there are total $8 \cdot 2^{\eta}+21$ atoms, among them $4 \cdot 2^{\eta}$ atoms are of valency $1,4 \times 2^{\eta}+12$ atoms are of valency 3,8 atoms are of valency 2 , and one atom is of valency 4 .

The number of atom-bonds in $\mathcal{M}_{52}$ is $8 \cdot 2^{\eta}+28$, by formula (1). According to the valencies of atoms incident with each atom-bond, there are five types $1 \sim 3$, $2 \sim 2,2 \sim 3,3 \sim 3$, and $3 \sim 4$ of atom-bonds in $\mathcal{M}_{52}$. Equivalently, the atom-bonds set of $\mathcal{M}_{52}$ is partitioned as $B\left(\mathcal{M}_{52}\right)=B_{1 \sim 3} \cup B_{2 \sim 2} \cup B_{2 \sim 3} \cup B_{3 \sim 3} \cup B_{3 \sim 4}$, where $B_{1 \sim 3}=\left\{\epsilon=a b \in B\left(\mathcal{M}_{52}\right) \mid v_{a}=1, v_{b}=3 \wedge v_{\epsilon}=2\right\}$ with $\left|B_{1 \sim 3}\right|=4 \cdot 2^{\eta}$,

$B_{2 \sim 2}=\left\{\epsilon=a b \in B\left(\mathcal{M}_{52}\right) \mid v_{a}=2, v_{b}=2 \wedge v_{\epsilon}=2\right\}$ with $\left|B_{2 \sim 2}\right|=4$,

$B_{2 \sim 3}=\left\{\epsilon=a b \in B\left(\mathcal{M}_{52}\right) \mid v_{a}=2, v_{b}=3 \wedge v_{\epsilon}=3\right\}$ with $\left|B_{2 \sim 3}\right|=8$,

$B_{3 \sim 3}=\left\{\epsilon=a b \in B\left(\mathcal{M}_{52}\right) \mid v_{a}=3, v_{b}=3 \wedge v_{\epsilon}=4\right\}$ with $\left|B_{3 \sim 3}\right|=4 \cdot 2^{\eta}+12$,

$B_{3 \sim 4}=\left\{\epsilon=a b \in B\left(\mathcal{M}_{52}\right) \mid v_{a}=3, v_{b}=4 \wedge v_{\epsilon}=5\right\}$ with $\left|B_{3 \sim 4}\right|=4$.

Analogously as in the case of porphyrin cored dendrimerI, $\mathcal{M}_{51}$, using the atom-bonds partition in formulae of the 
Banhatti polynomials from (7) and (8), it can be found that

$$
\begin{aligned}
B_{1}\left(\mathcal{M}_{52}, x\right)= & \left(4 \cdot 2^{\eta}+4\right) x^{8}+8 x^{11} \\
& +\left(4 \cdot 2^{\eta}+12\right) x^{14}+4 x^{17} \\
B_{2}\left(\mathcal{M}_{52}, x\right)= & 4 \cdot 2^{\eta} x^{8}+4 x^{8}+8 x^{15} \\
& +\left(4 \cdot 2^{\eta}+12\right) x^{24}+4 x^{35}, \\
H B_{1}\left(\mathcal{M}_{52}, x\right)= & 4 \cdot 2^{\eta} x^{34}+8 x^{16}+8 x^{61} \\
& +\left(4 \cdot 2^{\eta}+12\right) x^{98}+4 x^{145}, \\
H B_{2}\left(\mathcal{M}_{52}, x\right)= & 4 \cdot 2^{\eta} x^{40}+8 x^{16}+8 x^{117} \\
& +\left(4 \cdot 2^{\eta}+12\right) x^{288}+4 x^{625} .
\end{aligned}
$$

With respect to $x$, by taking the first derivative of these polynomials at $x=1$, we get the following K Banhatti indices of $\mathcal{M}_{52}$.

$\operatorname{TI}\left(\mathcal{M}_{52}\right)=\left\{\begin{array}{cll}88 \cdot 2^{\eta}+356 & , & \text { when } T I \text { is } B_{1}, \\ 128 \cdot 2^{\eta}+580 & , & \text { when } T I \text { is } B_{2}, \\ 528 \cdot 2^{\eta}+2372 & , & \text { when } T I \text { is } H B_{1} \\ 1312 \cdot 2^{\eta}+7020 & , & \text { when } T I \text { is } H B_{2} .\end{array}\right.$

Using relationships (9), (10), (13) and (14), Zagreb and forgotten indices of $\mathcal{M}_{52}$ are:

$$
\operatorname{TI}\left(\mathcal{M}_{52}\right)=\left\{\begin{array}{ccl}
40 \cdot 2^{\eta}+156 & , & \text { when } T I \text { is } Z_{1}, \\
48 \cdot 2^{\eta}+220 & , & \text { when } T I \text { is } Z_{2}, \\
208 \cdot 2^{\eta}+892 & , & \text { when } T I \text { is } H Z, \\
112 \cdot 2^{\eta}+452 & , & \text { when } T I \text { is } F .
\end{array}\right.
$$

\section{Porphyrin cored dendrimer-III}

Our seventh molecular graph $\mathcal{M}_{53}$ is the porphyrin cored dendrimer-III (PCD-III) of generation $G_{\eta}$ with $\eta \geq 1$ growth stage. $\mathcal{M}_{53}$ consists of eight similar branches and one central core. The central core contains 4 atoms of valency 2 , 20 atoms of valency 3, 1 atom of valency 4, and 40 atombonds as shown in Figure 7. In one branch of $\mathcal{M}_{53}$, there are $2^{0}+2^{1}+2^{2}+\ldots+2^{\eta}=2^{\eta+1}-1$ atoms, among them $2^{\eta}$ atoms are of valency 1 , and $2^{0}+2^{1}+2^{2}+\ldots+2^{\eta-1}=2^{\eta}-1$ atoms are of valency 3 . Therefore, in $\mathcal{M}_{53}$, there are total $16 \cdot 2^{\eta}+17$ atoms, among them $8 \cdot 2^{\eta}$ atoms are of valency $1,8 \times 2^{\eta}+12$ atoms are of valency 3,4 atoms are of valency 2 , and one atom is of valency 4 .

The number of atom-bonds in $\mathcal{M}_{53}$ is $16 \cdot 2^{\eta}+24$, by formula (1). According to the valencies of atoms incident with each atom-bond, there are four types $1 \sim 3,2 \sim 3$, $3 \sim 3$, and $3 \sim 4$ of atom-bonds in $\mathcal{M}_{53}$. Equivalently, the atom-bonds set of $\mathcal{M}_{53}$ is partitioned as $B\left(\mathcal{M}_{53}\right)=B_{1 \sim 3} U$ $B_{2 \sim 3} \cup B_{3 \sim 3} \cup B_{3 \sim 4}$, where

$B_{1 \sim 3}=\left\{\epsilon=a b \in B\left(\mathcal{M}_{53}\right) \mid v_{a}=1, v_{b}=3 \wedge v_{\epsilon}=2\right\}$ with $\left|B_{1 \sim 3}\right|=8 \cdot 2^{\eta}$,
$B_{2 \sim 3}=\left\{\epsilon=a b \in B\left(\mathcal{M}_{53}\right) \mid v_{a}=2, v_{b}=3 \wedge v_{\epsilon}=3\right\}$ with $\left|B_{2 \sim 3}\right|=8$,

$B_{3 \sim 3}=\left\{\epsilon=a b \in B\left(\mathcal{M}_{53}\right) \mid v_{a}=3, v_{b}=3 \wedge v_{\epsilon}=4\right\}$ with $\left|B_{3 \sim 3}\right|=8 \cdot 2^{\eta}+12$,

$B_{3 \sim 4}=\left\{\epsilon=a b \in B\left(\mathcal{M}_{53}\right) \mid v_{a}=3, v_{b}=4 \wedge v_{\epsilon}=5\right\}$ with $\left|B_{3 \sim 4}\right|=4$.

Analogously as in the case of porphyrin cored dendrimerI, $\mathcal{M}_{51}$, using the atom-bonds partition in formulae of the Banhatti polynomials from (7) and (8), it can be found that

$$
\begin{aligned}
B_{1}\left(\mathcal{M}_{53}, x\right)= & 8 \cdot 2^{\eta} x^{8}+8 x^{11} \\
& +\left(8 \cdot 2^{\eta}+12\right) x^{14}+4 x^{17} \\
B_{2}\left(\mathcal{M}_{53}, x\right)= & 8 \cdot 2^{\eta} x^{8}+8 x^{15} \\
& +\left(8 \cdot 2^{\eta}+12\right) x^{24}+4 x^{35}, \\
H B_{1}\left(\mathcal{M}_{53}, x\right)= & 8 \cdot 2^{\eta} x^{34}+8 x^{61} \\
& +\left(8 \cdot 2^{\eta}+12\right) x^{98}+4 x^{145}, \\
H B_{2}\left(\mathcal{M}_{53}, x\right)= & 8 \cdot 2^{\eta} x^{40}+8 x^{117} \\
& +\left(8 \cdot 2^{\eta}+12\right) x^{288}+4 x^{625} .
\end{aligned}
$$

With respect to $x$, by taking the first derivative of these polynomials at $x=1$, we get the following $\mathrm{K}$ Banhatti indices of $\mathcal{M}_{53}$.

$\operatorname{TI}\left(\mathcal{M}_{53}\right)=\left\{\begin{array}{cll}176 \cdot 2^{\eta}+324 & , & \text { when } T I \text { is } B_{1}, \\ 256 \cdot 2^{\eta}+548 & , & \text { when } T I \text { is } B_{2}, \\ 1056 \cdot 2^{\eta}+2244 & , & \text { when } T I \text { is } H B_{1} \\ 2624 \cdot 2^{\eta}+6892 & , & \text { when } T I \text { is } H B_{2} .\end{array}\right.$

Using relationships (9), (10), (13) and (14), we get Zagreb and forgotten indices of $\mathcal{M}_{53}$.

$$
\operatorname{TI}\left(\mathcal{M}_{53}\right)=\left\{\begin{array}{cll}
40 \cdot 2^{\eta}+140 & , & \text { when } T I \text { is } Z_{1}, \\
96 \cdot 2^{\eta}+204 & , & \text { when } T I \text { is } Z_{2}, \\
416 \cdot 2^{\eta}+828 & , & \text { when } T I \text { is } H Z, \\
224 \cdot 2^{\eta}+420 & , & \text { when } T I \text { is } F .
\end{array}\right.
$$

\section{Porphyrin Cored dendrimer-IV}

Our eighth and last molecular graph $\mathcal{M}_{54}$ is the porphyrin cored dendrimer-IV (PCD-IV) of generation $G_{\eta}$ with $\eta \geq 1$ growth stage. $\mathcal{M}_{54}$ consists of twelve similar branches and one central core. The central core contains 24 atoms of valency 3, 1 atom of valency 4, and 44 atom-bonds as shown in Figure 8. In one branch of $\mathcal{M}_{54}$, there are $2^{0}+2^{1}+2^{2}+\ldots+$ $2^{\eta}=2^{\eta+1}-1$ atoms, among them $2^{\eta}$ atoms are of valency 1 , and $2^{0}+2^{1}+2^{2}+\ldots+2^{\eta-1}=2^{\eta}-1$ atoms are of valency 3 . Therefore, in $\mathcal{M}_{54}$, there are total $24 \cdot 2^{\eta}+13$ atoms, among them $12 \cdot 2^{\eta}$ atoms are of valency $1,12 \times 2^{\eta}+12$ atoms are of valency 3 , and one atom is of valency 4 .

The number of atom-bonds in $\mathcal{M}_{54}$ is $24 \cdot 2^{\eta}+20$, by formula (1). According to the valencies of atoms incident 
with each atom-bond, there are four types $1 \sim 3,3 \sim 3$, and $3 \sim 4$ of atom-bonds in $\mathcal{M}_{54}$. Equivalently, the atombonds set of $\mathcal{M}_{54}$ is partitioned as $B\left(\mathcal{M}_{54}\right)=B_{1 \sim 3} \cup B_{3 \sim 3} \cup$ $B_{3 \sim 4}$, where

$B_{1 \sim 3}=\left\{\epsilon=a b \in B\left(\mathcal{M}_{54}\right) \mid v_{a}=1, v_{b}=3 \wedge v_{\epsilon}=2\right\}$ with $\left|B_{1 \sim 3}\right|=12 \cdot 2^{\eta}$,

$B_{3 \sim 3}=\left\{\epsilon=a b \in B\left(\mathcal{M}_{54}\right) \mid v_{a}=3, v_{b}=3 \wedge v_{\epsilon}=4\right\}$ with $\left|B_{3 \sim 3}\right|=12 \cdot 2^{\eta}+16$,

$B_{3 \sim 4}=\left\{\epsilon=a b \in B\left(\mathcal{M}_{54}\right) \mid v_{a}=3, v_{b}=4 \wedge v_{\epsilon}=5\right\}$ with $\left|B_{3 \sim 4}\right|=4$.

Analogously as in the case of porphyrin cored dendrimerI, $\mathcal{M}_{51}$, using the atom-bonds partition in formulae of the Banhatti polynomials from (7) and (8), it can be found that

$$
\begin{aligned}
B_{1}\left(\mathcal{M}_{54}, x\right) & =12 \cdot 2^{\eta} x^{8}+\left(12 \cdot 2^{\eta}+16\right) x^{14}+4 x^{17} \\
B_{2}\left(\mathcal{M}_{54}, x\right) & =12 \cdot 2^{\eta} x^{8}+\left(12 \cdot 2^{\eta}+16\right) x^{24}+4 x^{35}, \\
H B_{1}\left(\mathcal{M}_{54}, x\right) & =12 \cdot 2^{\eta} x^{34}+\left(12 \cdot 2^{\eta}+16\right) x^{98}+4 x^{145}, \\
H B_{2}\left(\mathcal{M}_{54}, x\right) & =12 \cdot 2^{\eta} x^{40}+\left(12 \cdot 2^{\eta}+16\right) x^{288}+4 x^{625} .
\end{aligned}
$$

With respect to $x$, by taking the first derivative of these polynomials at $x=1$, we get the following K Banhatti indices of $\mathcal{M}_{54}$.

$\operatorname{TI}\left(\mathcal{M}_{54}\right)=\left\{\begin{array}{cll}264 \cdot 2^{\eta}+292 & , & \text { when } T I \text { is } B_{1}, \\ 384 \cdot 2^{\eta}+524 & , & \text { when } T I \text { is } B_{2}, \\ 1584 \cdot 2^{\eta}+2148 & , & \text { when } T I \text { is } H B_{1}, \\ 3936 \cdot 2^{\eta}+7108 & , & \text { when } T I \text { is } H B_{2} .\end{array}\right.$

Using relationships (9), (10), (13) and (14), we have the following values for Zagreb and forgotten indices of $\mathcal{M}_{54}$.

$$
\operatorname{TI}\left(\mathcal{M}_{54}\right)=\left\{\begin{array}{lll}
120 \cdot 2^{\eta}+124 & , & \text { when } T I \text { is } Z_{1}, \\
144 \cdot 2^{\eta}+192 & , & \text { when } T I \text { is } Z_{2}, \\
624 \cdot 2^{\eta}+772 & , & \text { when } T I \text { is } H Z, \\
336 \cdot 2^{\eta}+388 & , & \text { when } T I \text { is } F .
\end{array}\right.
$$

\section{Geometric comparison and conclusion}

Topological indices are tools which are extensively useful for chemical analysis of molecular compounds. The results due to topological indices have remarkable significance in chemistry, engineering, bioscience and nanoscience applications. We considered numerous imperative molecular structures and computed their valency based topological indices by defining and computing four Banhatti polynomials. The obtained results are useful in forecasting several molecular properties of chemical compounds such as $\pi$-electron energy, medical configuration, boiling point and many more.

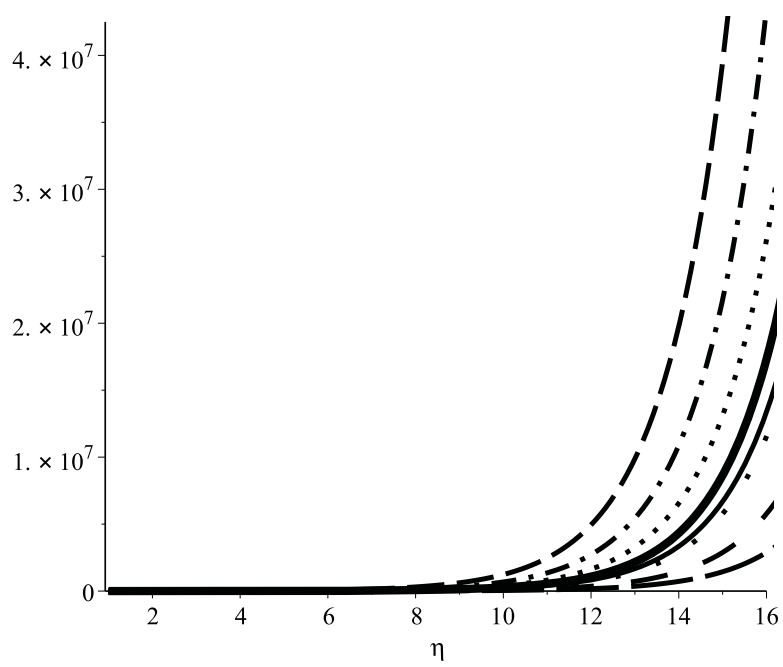

Figure 9 First K Banhatti index

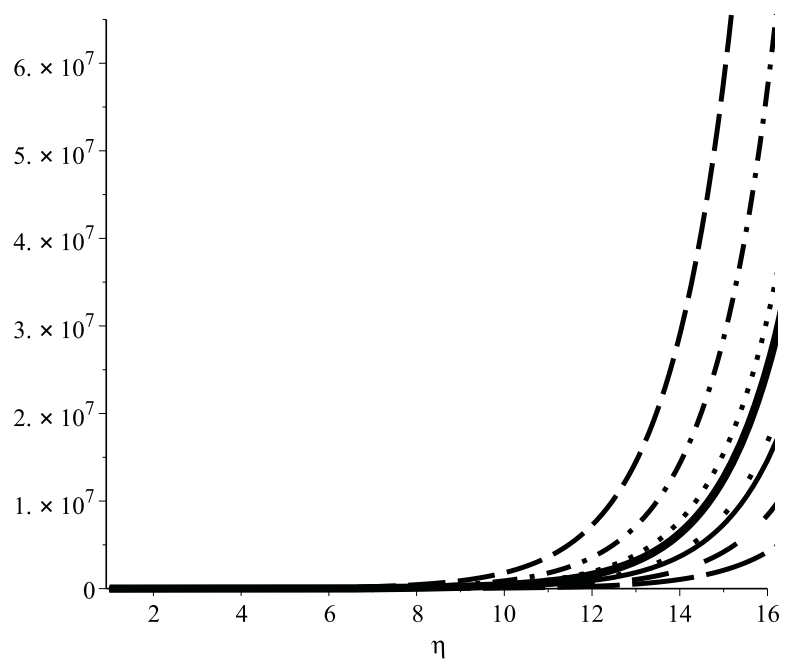

Figure 10 Second K Banhatti index

Using the technique of atom-bonds set partition of dendrimers in accordance with valencies and Banhatti polynomials, we investigated exact values for the first and second K Banhatti, K hyper Banhatti and Zagreb indices together with hyper Zagreb and forgotten indices. We separately plot values of each topological index obtained for eight dendrimers in Figure 9-16 to compare them geometrically. In each of these plots,

$\rightarrow$ thin solid curve is for poly (propyle) ethre imine (PETIM) dendrimer,

$\rightarrow$ short dotted curve is for poly ethylene amido amine (PETAA) dendrimer,

$\rightarrow$ short dashed curve is for porphyrin dendrimer $\left(D_{\kappa} P_{\kappa}\right)$,

$\rightarrow$ dash dotted curve is for zinc porphyrin dendrimer $\left(D P Z_{\eta}\right)$, 


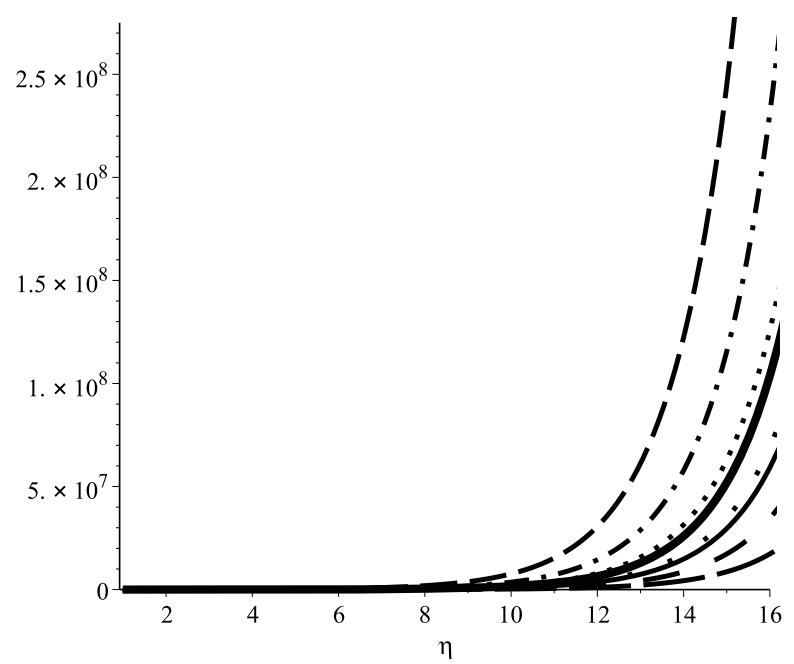

Figure 11 First K hyper Banhatti index

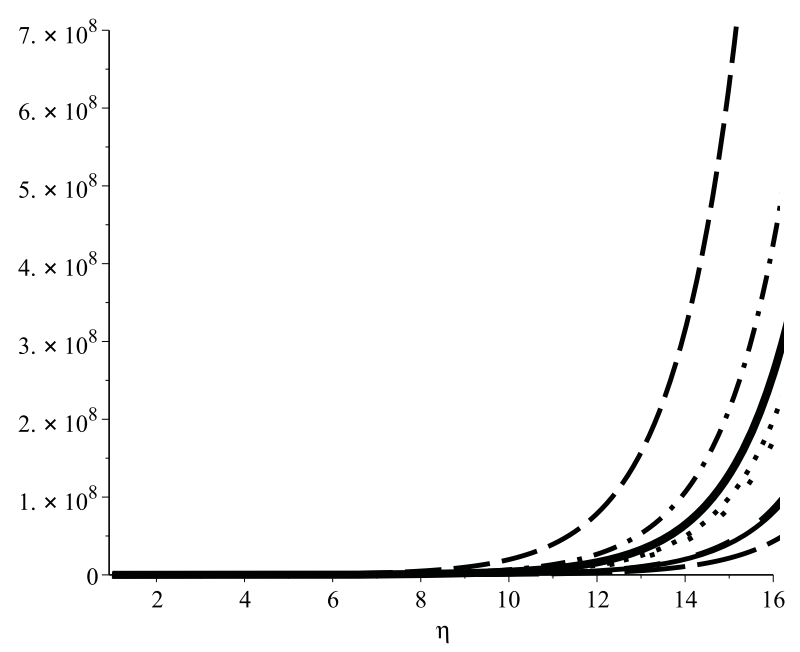

Figure 12 Second K hyper Banhatti index

$\rightarrow$ long dashed curve is for porphyrin cored dendrimer-I (PCD-I),

$\rightarrow$ space dashed curve is for porphyrin cored dendrimer-II (PCD-II),

$\rightarrow$ space dotted curve is for porphyrin cored dendrimer-III (PCD-III),

$\rightarrow$ thick solid curve is for porphyrin cored dendrimer-IV (PCD-IV).

Each plot depicts that the behavior of indices for every dendrimer is same for $\eta<10$. When $\eta$ is increasing, then $D_{\kappa} P_{\kappa}$ has greatest value of each index, whereas PCD-I has least value of each index. The dendrimer $D P Z_{\eta}$ has second greatest value of each index except of hyper Zagreb index, and for this index it preserves third least value as shown in the plot of Figure 15. It can also be observed that some

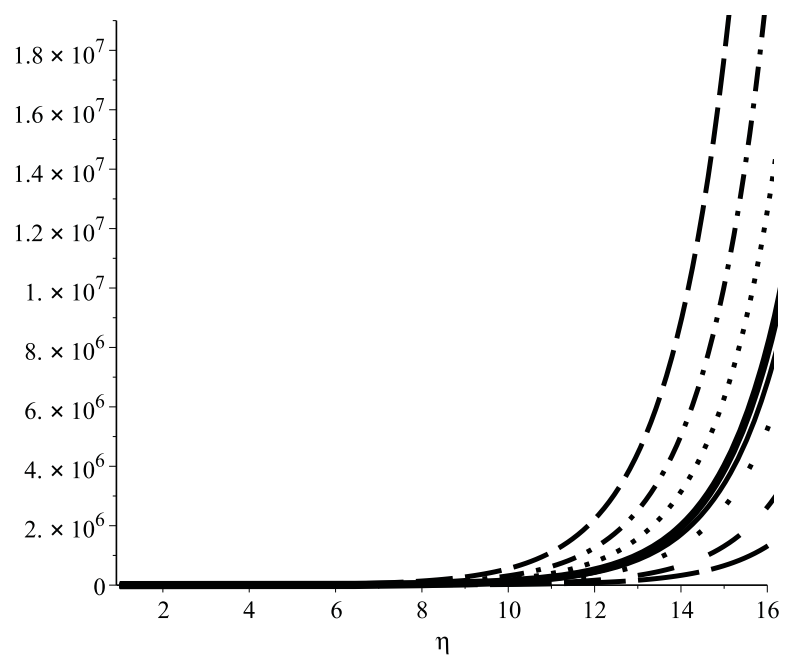

Figure 13 First Zagreb index

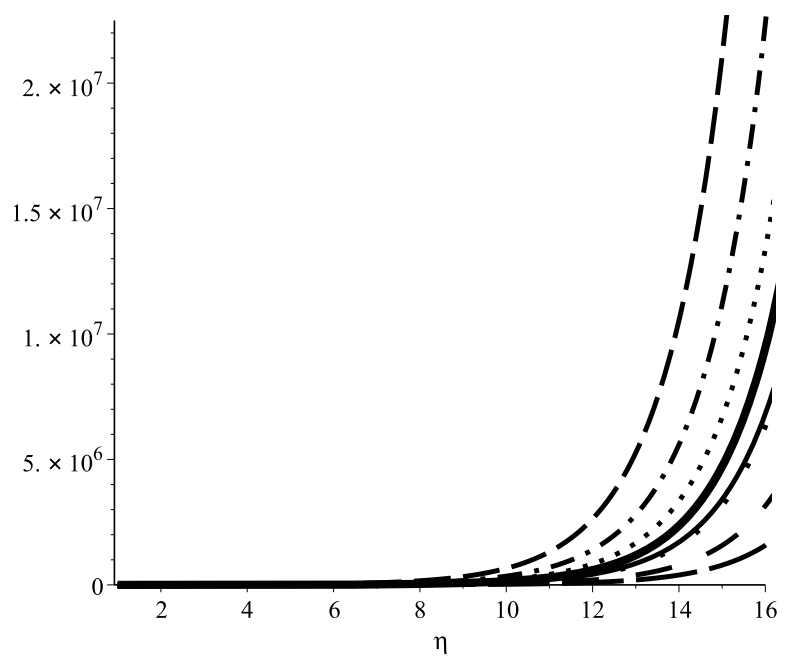

Figure 14 Second Zagreb index

of the dendrimers have almost coinciding and very nearest values of several indices, for instance:

- Plots in Figures 10, 11 and 14 show that PETIM and PCDIII have very nearest values of second $\mathrm{K}$ Banhatti, first $\mathrm{K}$ hyper Banhatti and second Zagreb indices;

- The plot in Figure 12 depicts that PETAA and PCD-III have very closest values of second $\mathrm{K}$ hyper Banhatti index, while this index receives almost coinciding values for PETIM and PCD-II;

- The plot in Figure 13 provides that the first Zagreb index behaved same for PETIM and PCD-I till $\eta<15$, and onward it gained very nearest values;

- Plots in Figures 15 and 16 show that the values of both the hyper Zagreb and forgotten indices coincide for PETIM and PCD-III. 


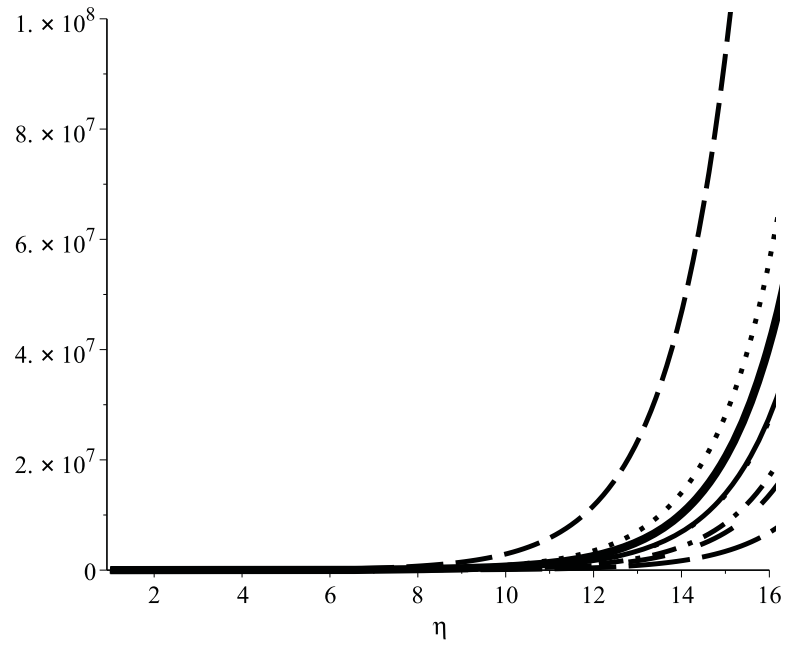

Figure 15 Hyper Zagreb index

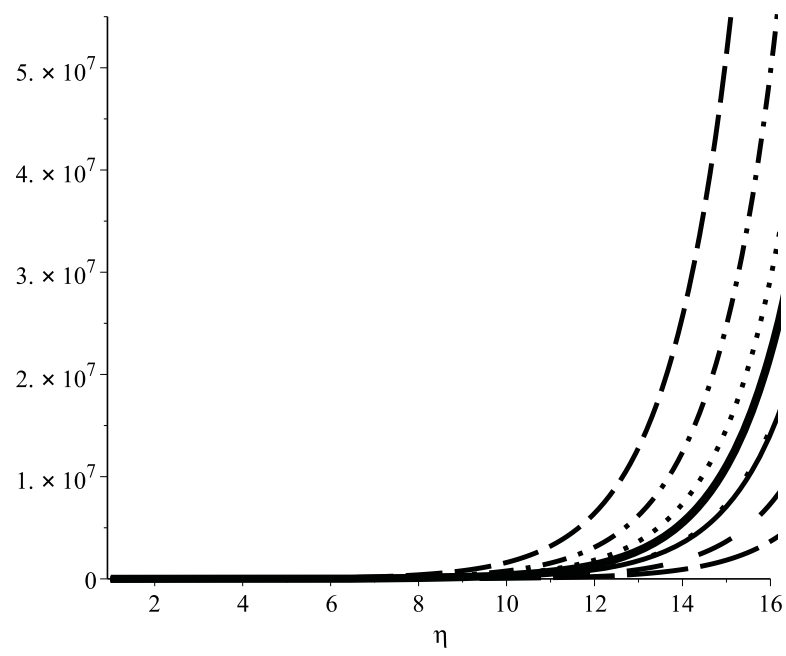

Figure 16 Forgotten index

Acknowledgments: The authors would like to express their sincere gratitude to the Natural Science Foundation for the Higher Education Institutions of Anhui Province of China (No. KJ2019A0875 and No. KJ2019A0876).

Conflict of interest: Authors state no conflict of interest.

\section{References}

[1] Alikhani S, Hasni R, Arif H. On the atom-bond connectivity index of some families of dendrimers. J Comput Theory of Nanosci. 2014;11:1802-1805.

[2] Anjum MS, Safdar MU. K Banhatti and K hyper Banhatti indices of nanotubes. Engineering Appl. Sci. Lett. 2019;2(1): 19-37.
[3] Arockiaraj M, Clement J, Balasubramanian K. Topological indices and their applications to circumcised donut benzenoid systems, kekulenes and drugs. Polycyclic Aromatic Compounds 2020;40(2):280-303.

[4] Arockiaraj M, Clement J, Balasubramanian K. Analytical expressions for topological Properties of Polycyclic Benzenoid Networks. J Chem. 2016;30:682-697.

[5] Asghar A, Rafaqat M, Nazeer W, Gao W. K Banhatti and K hyper Banhatti indices of circulant graphs. Int. J. Adv. Appl. Sci. 2018;5(5):107-109.

[6] Ashrafi AR, Mirzargar M. PI, Szeged and edge Szeged indices of an infinite family of nanostars dendrimers. Indian J Chem. 2008;47A:538-541.

[7] Balaban A, Klein D, Liu X. Graphitic vones. Carbon. 1994;32:357-359.

[8] Bashir Y, Aslam A, Kamran M, Qureshi MI, Jahangir A, Rafiq M, Bibi N, Muhammad N. On forgotten topological indices of some dendrimers structure. Molecules. 2017 May;22(6):867.

[9] Buhleier E, Wehner W, Vgtle F. "Cascade and NonskidChainlike" synthesis of molecular cavity topologies. Synthesis. 1978;2:155-158.

[10] Chartrand G, Zhang P. Itroduction to graph theory, New York: Tata McGraw-Hill Companies Inc; 2006.

[11] Diudea MV, Vizitiu AE, Mirzagar M, Ashrafi AR. Sadhana polynomial in nano-dendrimers. Carpath J Math. 2010;26:59-66.

[12] Furtula B, Gutman I. A forgotten topological index. J Math Chem. 2015;53:1184-1190.

[13] Goyal S, Garg P, Mishra VN. New composition of graphs and their Wiener indices. Appl. Math. Nonlinear Sci. 2019;4(1):163168.

[14] Gutman I, Trinajsti N. Graph theory and molecular orbitals. Total f-electron energy of alternant hydrocarbons. Chem Phys Lett. 1972;17:535-538.

[15] Kang SM, Zahid MA, Virk A, Nazeer W, Gao W. Calculating the degree-based topological indices of dendrimers. Open Chem. 2018;16:681-688.

[16] Kulli VR, Chaluvaraju B, Boregowda HS. Connectivity Banhatti indices for certain families of benzenoid systems. J. Ultra Chem. 2017;13(4):81-87.

[17] Kulli VR, On K Banhatti indices of graphs. J. Comp. Math. Sci. 2016;7(4):213-218.

[18] Kulli VR. On $\mathrm{K}$ hyper Banhatti indices and coindices of graphs. Int. J. Pure Algebra 2016;6(5):300-304.

[19] Kulli VR. On multiplicative K Banhatti and multiplicative K hyper Banhatti indices of V-Phenylenic nanotubes and nanotorus. Annals of Pure and Appl. Math. 2016;11(2):145-150.

[20] Kulli VR. Multiplicative K hyper Banhatti indices and coindices of graphs. Int J Math Arch. 2016;7(6):60-65.

[21] Liu J.-Bao, Wang C, Wang S, Wei B. Zagreb indices and multiplicative Zagreb indices of eulerian graphs. Bull Malays Math Sci Soc. 2019;42:67-78.

[22] Liu J.-Bao, Zhao J, Min J, Cao D. On the Hosoya index of graphs formed by a fractal graph. Fractals. 2019;27:1950135.

[23] Munir M, Nazeer W, Rafique S, Kang SM. M-polynomial and related topological indices of nanostar dendrimers. Symmetry. 2016;8:97.

[24] Rehman A, Nawaz M, Nazeer W, Gao W. K Banhatti and K hyper Banhatti indices of the line graphs of H-Pantacenic nanotubes. J. Prime Research in Math. 2018;14:62-73. 
[25] Shirdel GH, Rezapour H, Sayadi AM. The hyper Zagreb index of graph operations. Iranian J. Math. Chem. 2013;4(2): 213-220.

[26] Virk AR, Abbas T, Khalid W. Multiplicative topological descriptors of Silicon carbide. Appl. Math. Nonlinear Sci. 2019; 4(1):181-190.

[27] Wiener H. Structural determination of paraffin boiling points. J Am Chem Soc. 1947;69:17-20. 